\title{
A Proactive Strategy for Safe Human-Robot Collaboration based on a Simplified Risk Analysis
}

\author{
A. Sanderud ${ }^{1}$ T. Thomessen ${ }^{2}$ H. Osumi ${ }^{1}$ M. Niitsuma ${ }^{1}$ \\ ${ }^{1}$ Dept. of Precision Mechanics, Faculty of Science and Engineering, Chuo University, Tokyo, Japan. E-mail: \\ audun.sanderud@ppm.no, niitsuma@mech.chuo-u.ac.jp \\ ${ }^{2}$ PPM AS, Trondheim, Norway. E-mail: trygve.thomessen@ppm.no
}

\begin{abstract}
In an increasing demand for human-robot collaboration systems, the need for safe robots is crucial. This paper presents a proactive strategy to enable an awareness of the current risk for the robot. The awareness is based upon a map of historically occupied space by the operator. The map is built based on a risk evaluation of each pose presented by the operator. The risk evaluation results in a risk field that can be used to evaluate the risk of a collaborative task. Based on this risk field, a control algorithm that constantly reduces the current risk within its task constraints was developed. Kinematic redundancy was exploited for simultaneous task performance within task constraints, and risk minimization. Sphere-based geometric models were used both for the human and robot. The strategy was tested in simulation, and implemented and experimentally tested on a NACHI MR20 7-axes industrial robot.
\end{abstract}

Keywords: Safe Human-Robot Collaboration, Risk Field, Risk Analysis

\section{Introduction}

With the more open innovation model seen in the later years (Chesbrough, 2003), Small and Medium Enterprises (SMEs) have a growing importance in the industry. These types of companies require robotic equipment that is highly flexible, but also easy to use, both with regards to programming and operation. The most important approach to simple and flexible use of robots is through Human-Robot Collaboration (HRC)(Figure 1) and redundant robotics (Sanderud and Thomessen, 2014). A HRC system must be designed to fit the level of collaboration, but at any level the system should allow the operator to focus fully on his or her task, and not be concerned with where the robot is, or its current task. The robot should autonomously give the best assistance and avoid collisions at all times. A reliable safety strategy is therefore vital.

The most widespread protection strategy practiced in the industry is based on isolating robots from their surrounding environments (RIA/ANSI, 1999). While some HRC systems are commercially available, they have some major limitations.

RethinkRobotics (2013) have developed the Baxter system. Baxter is a double seven-axes arm, with a fully integrated control system. It can be installed in one hour and does not require any safety installations beyond the built-in safety system. But with only 2.3 $\mathrm{kg}$ payload per arm the work is limited to very light operations.

ABB have introduced the SafeMove system which is designed to bring the operator closer to the industrial robot (Behnisch, 2008). SafeMove operate with zones in which the operator can move safely, and allows a more efficient use of the robot. The robot will automatically slow down as the operator approaches, and go to a full stop if the operator is too close.

The SMErobots ${ }^{T M}$ initiative has done extensive research on, and developed systems to simplify both the industrial level programming and safety issues related 


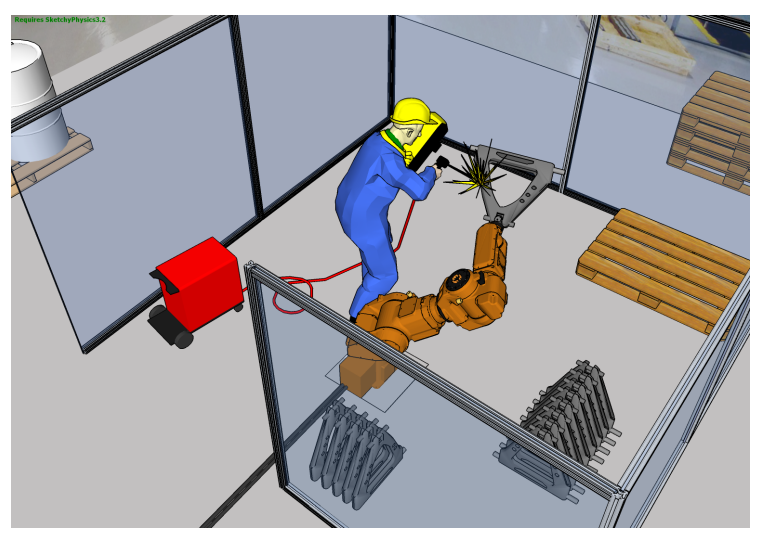

Figure 1: A collaboration between a human and a robot on a welding operation.

to industrial robot installations (SMErobotTM, 2013).

MRK-Systeme (2014) have a commercially available safety system for selected low payload robots. The system includes a capacitive cover for the robot, ensuring a full stop if the operator comes in contact with it. Moreover, the current safety standards allow very limited human-robot collaboration (RIA/ANSI, 1999).

A new standard covering collaborative robots is currently under development. The standard will allow closer human-robot collaboration, given that a set of performance control methods are implemented. Separation monitoring is one of these performance control methods. This is a system which at all times ensures that the robot manipulator is at a certain distance from the human operator to avoid injuries. Such a system will require an advanced sensor system and algorithms to reconfigure the robot manipulator based on sensor readings. With separation monitoring in place, a company can do highly complex human-robot collaborative tasks, reducing some of the pressure on programming of the robot prior to the operation. Also larger enterprises, such as the car manufacturing industry, can benefit from an efficient separation monitoring system. Just imagine a production line with tens of robots, and an operator walking amongst them, supervising and making adjustment while in full operation. The challenge is however not to compromise the productivity of the robot. A reasonable path-planning or real-time control of the robot is therefore an important aspect.

Most contributions towards collision avoidance with redundant robots are based on static or kinetostatic images. Systems finding a collision-free joint space path (Latombe and Barraquand, 1991), and problems that require maintaining end-effector constraints throughout the path (Stilman, 2007; Gupta, 2005; Berenson and Kuffner, 2011) have been explored. It is usually distinguished between problems where a single goal is specified (Ahuactzin and Gupta, 1999) and problems where the entire end-effector path is predetermined (Guo and Hsia, 1990; Oriolo et al., 2002). Applying this, Lacevic and Rocco (2010) presented an approach using Danger Fields based on a kinetostatic image of the current situation. Mainprice and Berenson (2013) used workspace occupancy predictions based on an articulated motion library for the human. A system anticipating human activities, and selecting the correct response from the robot was presented by Koppula and Saxena (2013). Zhang and Wang (2004) and Petrič and Žlajpah (2013) both present high performance systems that avoid collisions by enabling evasive maneuvers if a danger is detected. Balan and Bone (2006) presented an efficient human collision avoidance system. The system used prediction models on both the robot and the human to reduce the effect of non-instantaneous response time. In both systems, however, the task performance is easily compromised. Kulić and Croft (2006) describe a safety system based on a danger index. The system uses the danger index in a real-time trajectory generator to re-plan its path if a danger threshold is exceeded. These systems are all reactive and will manipulate the robot's path strictly to avoid an imminent collision. That is, when it is in a dangerous situation.

In our approach, we want to avoid the possible dangerous situation all together. We overcome this by building a cubic map of the work cell based on the risk associated with every point in space. The risk associated with a certain pose for the robot is calculated based on its occupied space. The robot will be enabled with an awareness of the risk related to its current pose, and planned path. If the risk is too high to accept, the robot has the chance to re-plan its path, or change its current pose. Based on this it can be more "cautious" if it is forced to operate in a highly risky area, e.g. move slower, make sound- or light signals or give vibrotactile feedback to the operator (Thomessen and Niitsuma, 2013). Vibrotactile feedback is researched to help in teleoperations, and shows a promising effect to deliver important information to the operator (Galambos, 2012; Muramatsu et al., 2013). This can easily be adapted to pass information from the robot system to the operator about the current risk, enabling more system awareness. Most importantly, by being proactive, the approach aims to reduce the number of dangerous situations to avoid, by acquiring knowledge about them at an early stage. The research of Hoffman et al. (2013) show how important trust in automation is, and how trust in machines can be related to interpersonal trust. An important feature to maintain trust is to avoid unexpected behavior by the robot. Avoiding the situations where evasive maneuvers are necessary could help enhance this important trust. A simplified 
risk model was implemented and experimentally tested on a NACHI MR20 7-axes industrial robot. The results illustrate the robots ability to avoid high risk areas in the workspace.

\section{Risk Analysis}

One of the most fundamental attributes of a human regarding safety is the ability to make a judgment of risk (Slovic, 1987). Different people accept different levels of risk in their daily routines at work and at home. All day, in every situation, there is a risk present. In a simple activity like crossing the road, we observe the frequency and speed of the cars, the width of the road and so on. We use these data, compare it to our physical attributes and experience. The data goes through numerous psychological and cognitive processes and tell us whether to cross or not. If the parameters make us not cross, we might walk along the road to search for a change in some of the parameters. Along the road, authorities may have implemented measures like traffic lights or zebra stripes to create safer crossings. This is all based on a risk analysis, and what level of risk we are willing to accept.

The goal of any safety strategy is to reduce the risk. In this research we use the definition of risk, set by NORSOK (2001). In NORSOK z-013N risk is defined as a combination of the probability of an event, and the consequence of the event. Further, a risk analysis is the process of using available information to identify possible accidents and estimate the risk. The consequence is a complex entity itself. The consequences of an accident can impact several aspects of a company. These include reduced production, personal injury, material damage and the company's reputation. Although companies might prioritize the different consequences differently, avoiding personal injury is generally considered the worst consequence. Most importantly in an isolated case of a robot cell is to prioritize avoiding human injury over reduced production and material damage. To reduce the risk, one can implement what is defined as Risk Reducing Measures (RRMs). In other words, a safety strategy involves a number of RRMs.
In a HRC context, we identify four approaches to risk reducing measures (Table 1). Either focused on the human, or on the robot, and, either reducing the likelihood, or the consequence. RRMs aimed at the human includes fences, and light- and sound signals. These actions will remind the human of the danger associated with approaching a moving robot. Thus making it less likely that the human approaches the robot. This is the most commonly used RRM today (RIA/ANSI, 1999). The second common approach is aimed at the robots. These include designing the robots to be less harmful, like RethinkRobotics (2013) or the KUKA lightweight Robot (KUKA, 2014). The system provided by MRKSysteme (2014) ensures that if an accident should occur, it will have very little consequence in a safety perspective. Lightweight and slow robots will reduce the consequence of an accident. Another RRM is to implement control algorithms to automatically avoid a human, like in the research of Lacevic and Rocco (2010), Petrič and Žlajpah (2013) and Kulić and Croft (2006). These avoidance algorithms would reduce the likelihood for an accident. The last RRM approach is likely never used in robotics. A RRM focused on the human to reduce the consequence of an accident would imply equipping the human with helmet, armor and other protective gear. Although a common approach in many other activities, it is extremely rare in robotics. A safety system will often be a combination of several RRMs. These are normally structured to be sequential based on which of the aforementioned consequences have the highest priority. Regarding only productivity and personal injury, it is obvious that preventing personal injuries has a higher priority. The robot focused consequence reducing RRMs will effectively prevent personal injury, but might cause an all new consequence; reduced productivity. This RRM should thus be a "last resort"-RRM. Table 2 lists a desired priority of RRMs. A proactive system could maintain the productivity to a greater extent than a reactive system. While an automatic emergency stop will reduce the productivity, it is an important RRM to utterly ensure safe human robot collaboration.

Our strategy for safe human-robot collaboration is thus to create a proactive safety strategy by enabling

Table 1: Risk Reducing Measures for Robotic Systems

\begin{tabular}{ccc}
\hline & Reduce Likelihood & Reduce Consequence \\
\hline Human Focused & Fences and barriers & Protective gear e.g. \\
& Light and Sound signals & Helmet and armor \\
Robot Focused & Obstacle avoidance systems & Lightweight and slow robots \\
& Proximity based emergency stop & Contact detection \\
\hline
\end{tabular}


Table 2: Priority for Risk Reducingf Measures

\begin{tabular}{cc}
\hline Priority & Risk Reducing Measure \\
\hline 1 & Proactive avoidance systems \\
2 & Reactive avoidance systems \\
3 & Proximity based emergency stop \\
4 & Contact based emergency stop \\
\hline
\end{tabular}

the robot with a sense of risk. Not only to enable a control algorithm to reduce the risk, but to communicate to the operator the current risk level. The operator and robot will thus have a common awareness of the present risk. In order to do this, we observe human activity through time. The nature of the human activity in a point in space provides the risk associated with that point. The resulting map serves as a memory for the robot of the risk associated with working or moving in different areas in its workspace.

To calculate the risk associated with a point in space at a given time, a risk analysis is necessary. As aforementioned, risk is defined as the correlation between likelihood and consequence. A risk analysis is therefore divided into a likelihood analysis, and a consequence analysis.

\subsection{Consequence Analysis}

Analyzing the consequence of an accident is a very challenging task that many have already researched. Haddadin et al. (2009a) and Haddadin et al. (2009b) investigated and identified different contact scenarios in a Human-Robot related accident. They identify the five contact scenarios constrained, partially constrained and unconstrained impact, clamping and secondary impact. The last often caused by one of the others, and might even be more severe. Some of these scenarios were tested in a crash-test during which contact forces, neck torques and other relevant data were collected. The data classifies the severity of the impacts. Haddadin and Albu-Schaffer (2008) also investigated injuries caused by sharp tools on the robot, and developed a reactive avoidance system. A natural approach to measuring the danger is presented by Kulić and Croft (2006). They compute a danger index based on factors affecting the impact force during a potential collision. A simplified consequence analysis will therefore include the parameters Limb velocity $\left(v_{t}\right)$ and Limb type factor $\left(L_{f}\right)$, as seen in (1). A simplified model of critical areas of the human body is developed based upon Haddadin et al. (2009a) and Haddadin et al. (2009b). Where the torso and head are the most critical and, the hands and feet are the least critical area (Figure 2). Other factors that could be implemented are the robots velocity and tool.

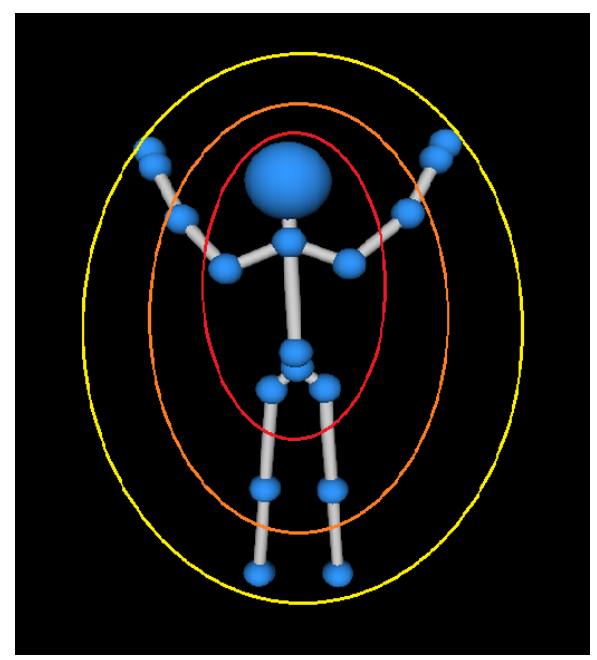

Figure 2: Map of limb factor areas used in the consequence analysis. Red indicate a higher limb factor, yellow a lesser.

$$
C=L_{f}\left(1+v^{2}\right)
$$

\subsection{Likelihood Analysis}

The likelihood analysis is in this research based on the visit frequency at a given point in space. It will therefore evolve, according to the humans motion and activity. While there are several examples on human task recognition and classification (Koppula and Saxena, 2013; Mainprice and Berenson, 2013), a great part of the articulated human motion is not part of a task. Movement like scratching the head, checking the time, turning to see what that sound was, is generally difficult to classify. This motion is most of the time unpredictable and inaccurate. Moreover, a tracked limb of the human will seldom take the exact same path during its next pass. The likelihood is therefore calculated for the limb and its close proximity up to a maximum distance, $\rho$, from the limb. This factor will also compensate for smaller errors in sensor data readings of limb positions. The resulting proximity factor, $\kappa$, is found by:

$$
\kappa=\left\{\begin{array}{cl}
\frac{\rho-\delta}{\rho} & \text { if } \delta \leq \rho \\
0 & \text { otherwise }
\end{array}\right.
$$

Where $\delta$ is the distance from a point in space to the human limb, and $\rho$ is the maximum distance from a limb where the risk should be computed. 
We model the contribution from the likelihoods as an exponentially decaying positive feedback with a reduction factor, $\gamma$,

$$
y_{i}=\kappa C+y_{i-1} \gamma
$$

where $C$ is the result of the consequence analysis and $i$ is the iteration.

\section{Risk Field}

Let $\mathrm{T}$ be a human limb whose position and velocity are defined by the vectors $\mathbf{r}_{t}=\left(\begin{array}{lll}x_{t} & y_{t} & z_{t}\end{array}\right)^{T}$ and $\mathbf{v}_{t}=$ $\left(\begin{array}{lll}v_{t x} & v_{t y} & v_{t z}\end{array}\right)^{T}$. The $\delta$ from (2) is then $\delta=\left\|\mathbf{r}-\mathbf{r}_{t}\right\|$, where $\mathbf{r}=(x y z)^{T}$ is an arbitrary point in space. The velocity vectors magnitude is denoted $v_{t}=\left\|\mathbf{v}_{t}\right\|$. From our consequence analysis (1) and risk analysis (3) we can quantify the risk related to a single point in space. The risk field created by a single moving human limb at time $i, R F(\mathbf{r})_{i}$, is then defined by (4) with $\kappa$ from (5).

$$
\begin{array}{r}
R F(\mathbf{r})_{i}=\kappa L_{f}\left(1+v_{t}^{2}\right)+\gamma R F(\mathbf{r})_{i-1} \\
\kappa=\left\{\begin{array}{cl}
\frac{\rho-\left\|\mathbf{r}-\mathbf{r}_{t}\right\|}{\rho} & \text { if }\left\|\mathbf{r}-\mathbf{r}_{t}\right\| \leq \rho \\
0 & \text { otherwise }
\end{array}\right.
\end{array}
$$

While multiple limbs may pose a risk at $\mathbf{r}$, it is necessary to accumulate the risk posed by every limb. The risk field is thus expanded and derived by super positioning. $R F(\mathbf{r})_{i}$ is then the sum of the risk posed by all spheres for every limb (6). Each limb and sphere will have a specified $\rho_{t}$ and $L_{f, t}$. Each sphere of the sphere-based geometry of the human is used to calculate the risk. The total number of spheres, $l$, and their radii, $\rho_{i}$, should be selected to best model the human body.

$$
\begin{aligned}
& R F(\mathbf{r})_{i}=\sum_{t=1}^{l} \kappa_{t} L_{f, t}\left(1+v_{t}^{2}\right)+\gamma R F(\mathbf{r})_{i-1} \\
& \kappa_{t}=\left\{\begin{array}{cl}
\frac{\rho-\left\|\mathbf{r}-\mathbf{r}_{t}\right\|}{\rho_{t}} & \text { if }\left\|\mathbf{r}-\mathbf{r}_{t}\right\| \leq \rho_{t} \\
0 & \text { otherwise }
\end{array}\right.
\end{aligned}
$$

The field $R F(\mathbf{r})_{i}$ is by definition a scalar field. Nevertheless, a vector field can easily be constructed upon it using its gradient (7).

$$
\overrightarrow{R F}(\mathbf{r})_{i}=R F(\mathbf{r})_{i} \frac{\nabla R F(\mathbf{r})_{i}}{\left\|\nabla R F(\mathbf{r})_{i}\right\|}
$$

The $\overrightarrow{R F}(\mathbf{r})_{i}$ vector is anchored in $\mathbf{r}$ and with the direction of $\nabla R F(\mathbf{r})_{i}$. Its magnitude is set by the risk level in $\mathbf{r}, R F(\mathbf{r})_{i}$.

\section{Risk Field-Based Control}

\subsection{Control Principals}

The control method bares many similarities to the classic potential field model (Khatib, 1985) or the avoidance strategy of Zlajpah and Nemec (2002). The major difference lies in the design of the potential field. While the classical potential field model regards a single static image of the obstacle, our model regards a variety of parameters for the object, resulting in a quantified risk measure. Further, the risk associated with areas in the workspace is kept in the control systems memory. Allowing the system to avoid risky areas, even though an obstacle is not detected there at the current time.

In the avoidance strategy of Zlajpah and Nemec (2002), an avoidance vector $\Delta \mathbf{p}_{a}$ is placed at the critical points of the robot, directly away from the object. The avoidance vector is then mapped to joint space via the transposed Jacobian. A critical point is defined by the proximity of an object (Figure 3). The resemblance to the virtual impedance approach (Tsuji et al., 1997; Nakabo and Ishikawa, 1998) is also clear. In this method does the penetration depth in the critical area determine the velocity and acceleration of the avoidance vector. Much resembling the real-life impedance control, considering physical contact between the robot and an object. The difference to our method is quite clear. The direction and magnitude of the avoidance vector is selected directly from the risk field. A predetermined number of points on the robot is selected to be examined for potential risk. Any observed point pose a higher risk than accepted, will have an avoidance vector with a magnitude and direction set by the risk field (Figure 4).

From the safety perspective, our method is inspired by the research of Kulić and Croft (2006) and Lacevic et al. (2013). However, Kulić and Croft (2006) do not consider task constraints, and thus neglects loss productivity as a consequence. Although it is correct to assume that reduced productivity is a less important consequence than human injury or material damage. The method thus holds from a pure safety perspective, but not a human-robot collaborative perspective. The method presented by Lacevic et al. (2013) considers task constraints, but their Danger Field only observes and analyzes the motion of the robot. The humans motion and movement patterns are not included. The system is reactive, which may cause more unexpected maneuvers than necessary, reducing the operators trust 


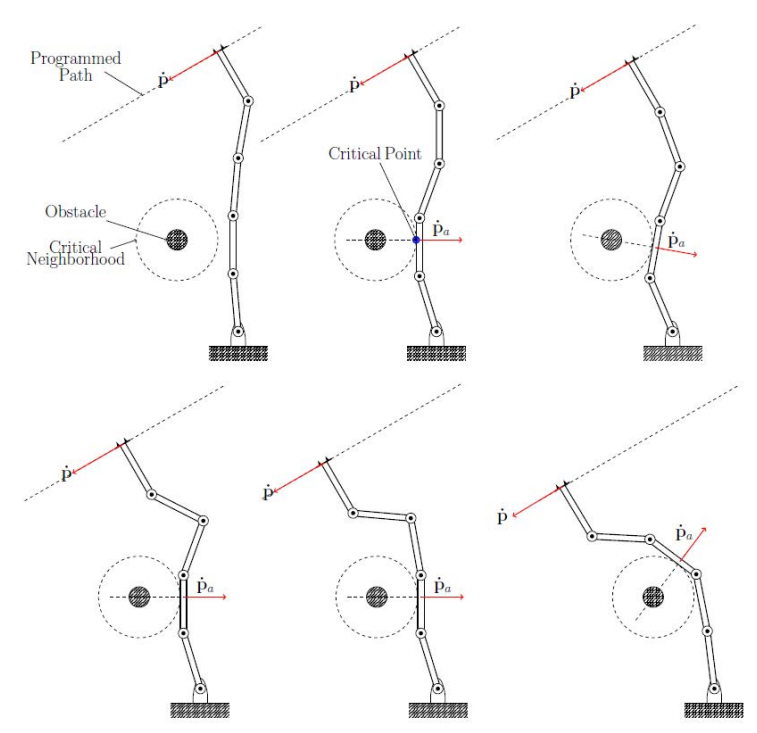

Figure 3: Obstacle avoidance strategy on a 5-axes planar arm. in the system. With our method's memory of previous human activity, the robot can plan its path from the risk field, resulting in smoother motions. Even with a reactive response to the risk field, the system will be proactive because of the history that lies in the map. Further, the robot task velocity can be controlled based on the risk field and level of accepted risk. This awareness of risk for the robot can then be communicated to the human, further enhancing the trust.

\subsection{Kinematic Control of Redundant Robot}

The redundancy resolution and control method in this paper is an extension of the Null Space Projection Method where a secondary task vector is projected in the Null Space of the robot (Patel and Shadpey, 2005). The resulting velocity vector $\dot{\mathbf{q}}_{\aleph}$ is added to the primary task's joint velocity vector $\dot{\mathbf{q}}_{T}$. The primary task joint velocities is obtained from the primary task cartesian velocities via the transposed Jacobian. The classical Null Space formulation, at a velocity level, is stated in (8), where $\vartheta$ is the joint velocities of the secondary task.

$$
\begin{array}{r}
\dot{\mathbf{q}}=\dot{\mathbf{q}}_{T}+\dot{\mathbf{q}}_{\aleph}=J_{e}^{\dagger} \dot{\mathbf{p}}_{T}+\left(I-J_{e}^{\dagger} J_{e}\right) \vartheta \\
\vartheta=J_{e}^{\dagger} \dot{\mathbf{p}}_{S}
\end{array}
$$

This method alone will not manipulate the robots end effector, only produce self motion. The Virtually Extended Null Space formulation was therefore used. The method is described in previous work of the authors (Sanderud, 2012; Sanderud et al., 2014), it will however be recalled in the remainder of this section.

In the classical Null Space method with a 7-axes robot, the dimension of the redundancy $r$, and also the null space, is $r=n-m$, where $\mathrm{n}$ is the dimensions of task space, and $m$ the dimensions of joint space. Then the kinematic chain of the robot is virtually extended with a parallel to the cartesian space. For a 7 -axes robot, that mens that $r=(n+m)-m=n=7$. A projection in the Null Space of this virtually extended joint space will thus provide a 7-dimensional motion, 6 of which are parallel to task space. The displacement in these 6 axes $\left(\dot{\mathbf{q}}^{v}\right.$ from (9)) is defined as $\dot{\mathbf{p}}^{v}$, and is added to the task velocities $\dot{\mathbf{p}}_{\mathbf{T}}$.

$$
\left[\begin{array}{c}
\dot{\mathbf{q}}_{s} \\
\dot{\mathbf{q}}^{v}
\end{array}\right]=\left(I-J_{W}^{\dagger} J_{W}\right)\left[\begin{array}{l}
\vartheta \\
\mathbf{0}
\end{array}\right]
$$

To keep within task constraints, a weighting matrix,$W$, is introduced in to the virtually extended 
part of the new Jacobian (10). The weighting matrix is a diagonal positive-definite matrix of the form $W=\operatorname{diag}\left[\begin{array}{llll}w_{1} & w_{2} & \ldots & w_{n}\end{array}\right]$

$$
J_{W}=\left[\begin{array}{c}
J_{e} \\
J_{v} W
\end{array}\right]
$$

With this, the joint velocities $\dot{\mathbf{q}}$ are then calculated from (11).

$$
\left[\begin{array}{c}
\dot{\mathbf{q}} \\
\dot{\tilde{\mathbf{q}}}^{v}
\end{array}\right]=\left[\begin{array}{c}
J_{e}^{\dagger}\left(\dot{\mathbf{p}}_{T}+\dot{\mathbf{p}}^{v}\right) \\
\mathbf{0}
\end{array}\right]+\left(I-J_{W}^{\dagger} J_{W}\right)\left[\begin{array}{l}
\vartheta \\
\mathbf{0}
\end{array}\right]
$$

Regarding our industrial robot arm, a predefined set of points along the arm is to be examined for a present risk. The points on the manipulator with a present risk receive an avoidance vector $\Delta \mathbf{p}_{S}$. From section 3 we can use $\Delta \mathbf{p}_{S}=k_{p} \overrightarrow{R F}(\mathbf{r})_{i}$ for corresponding points in space, where $k_{p}$ is a real positive parameter. By dividing with the sample time, $\Delta T, \Delta \mathbf{p}_{S}$ becomes the velocity $\dot{\mathbf{p}}_{s}$ that governs the evasive maneuver. The joint velocities can be calculated via the pseudo inverse for the respective points along the kinematic chain. $\vartheta$ can then be computed as the sum of the joint velocities, calculated at all points of interest (12). Where $k_{v}=$ $k_{p} / \Delta T$.

$$
\vartheta=\sum_{S} J_{s}^{\dagger} \dot{\mathbf{p}}_{s}=\sum_{S} k_{v} J_{s}^{\dagger} \overrightarrow{R F}(\mathbf{r})_{i}
$$

The system structure is based on an interface to the NACHI FD11 controller, manipulating the data packages sent between the Main board and the servo board. The native stability and dynamics control of the NACHI controller is thus utilized. The system structure is further as described in Figure 5.

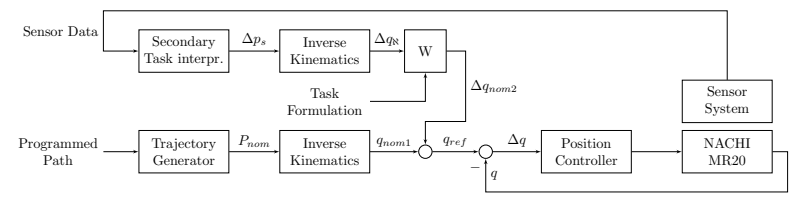

Figure 5: System structure with the secondary task in an external feedback loop.

Although the control scheme used in this study is the virtually extended null space, the information that lies in the risk field can generally be used with other schemes to. That is, both for real time collision avoidance control, and path planning, prior to, and during operation. Takimoto et al. (2014) presents a way to predict human goal location when in motion, based on a visit frequency map. Implementing this or similar strategies could also provide a predictive model, based on the likelihood analysis.

\section{Simulations}

\subsection{Simulation Setup}

The process of map design was tested through a two part simulation. The first to investigate the impact in the quantified risk in different situations. The second part to investigate how the risk is degenerated over time. Both parts include four limbs; two hands, and two heads, each of which either move slowly, or fast (Figure 6). The limbs were moved in a circular motion with constant speed throughout the first part (Figure 7 ). In the second part all limbs were removed, and the risk was slowly degenerating (Figure 8).

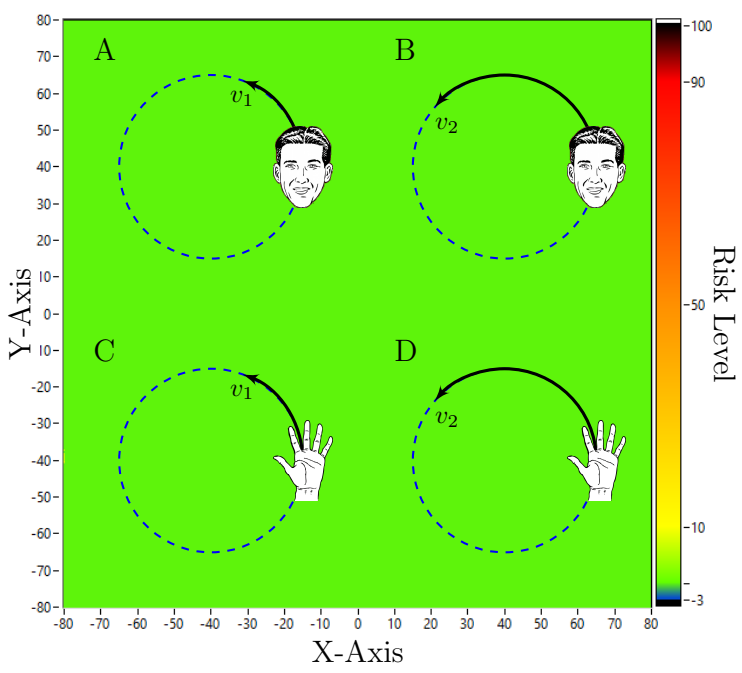

Figure 6: Simulation setup.

In both simulations parameters were selected to get results in a couple of seconds. The design parameters should be chosen carefully to get the desired map behavior. For this simulation, the following parameters were used: $\gamma=0.996, \quad L_{f}=\left[\begin{array}{llll}1 & 1 & 0.5 & 0.5\end{array}\right], \quad \rho=300 \mathrm{~mm}$, $v_{t}=\left[\begin{array}{llll}1 & 0.5 & 1 & 0.5\end{array}\right] \mathrm{m} / \mathrm{s}$.

\subsection{Simulation Results}

In the first part of the simulation it is clear that the area frequently occupied by the fast moving head is the most risky area for the robot the operate. A collision with a humans head must be avoided. While it is also moving fast, it would be less likely that the robot would be able to do a successful evasive maneuver, should the human head be detected in using an obstacle avoidance system. Conversely, it can be seen that the space less frequently occupied by the slow moving hand is barely 
considered risky. A slow moving hand can more easily be avoided by the robot, if required. Although to be avoided, but should a collision between the robot and a human hand occur the potential outcome is significantly less severe than a collision with the head.

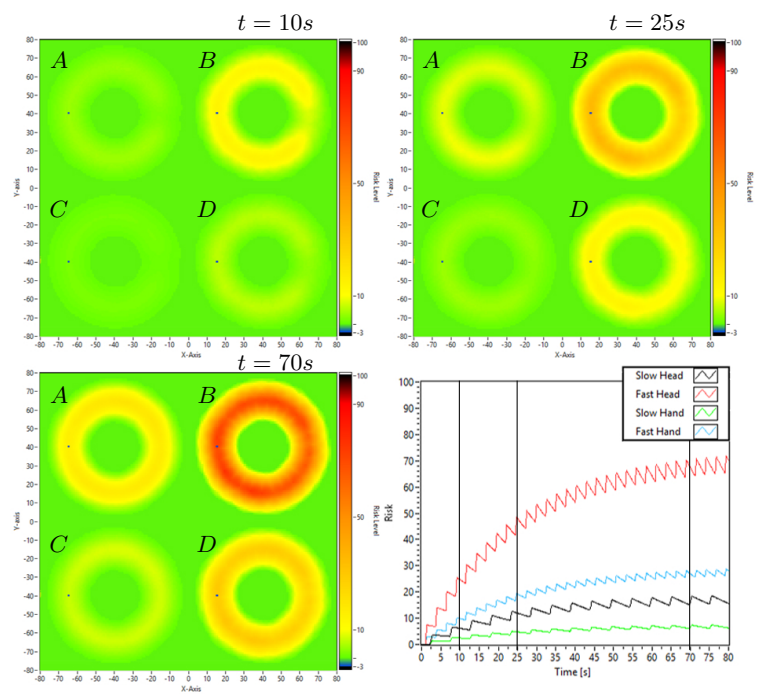

Figure 7: Simulated human activity with constant visit frequency over time. $\mathrm{A}=$ Slow Head, $\mathrm{B}=$ Fast Head, $\mathrm{C}=$ Slow Hand, $\mathrm{D}=$ Fast Hand. The lines on the graph indicate sample times

The last simulation shows that how the risk is degenerated over time. With the likelihood analysis (3), the risk will drop faster in the beginning, but keep a lower risk longer. Other approaches to get a different deterioration can be selected based on e.g. the task. With this approach it is suggested that the likelihood of a limb reentering the space it just left is low, while the risk should be degenerated slower over time. It is nonetheless clear that if a limb has been occupying by point in space in the past, there will always be a certain risk it will do it again.

\section{Experiments}

\subsection{Experimental setup}

For the experiment, a NACHI MR20 7-axes industrial robot was used with a NACHI FD11 controller. The controller was interfaced with an in-house system allowing manipulation of the controller's internal communication. An external PC running LabVIEW was used to develop and execute the code. The object was simulated in the LabVIEW code to ensure a predictable risk field for evaluation purposes.

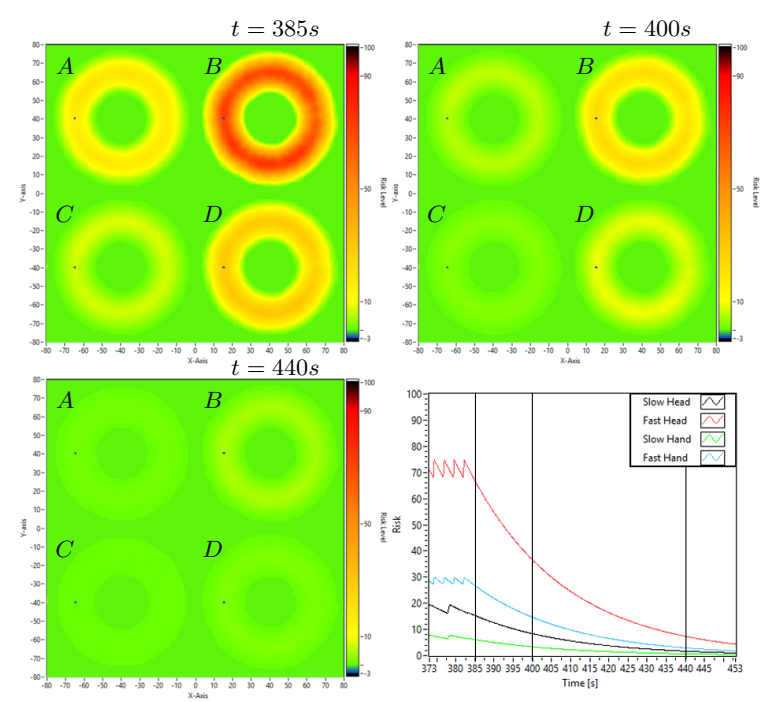

Figure 8: Degeneration of risk over time. $\mathrm{A}=\mathrm{Slow}$ Head, $\mathrm{B}=$ Fast Head, $\mathrm{C}=$ Slow Hand, $\mathrm{D}=$ Fast Hand. The lines on the graph indicate sample times

\subsection{Results}

The purpose of the experiments was to investigate how the robot would respond to the risk field. The robot was programmed to move in a straight line, from $P_{1}$ to $P_{2}$ (green line in Figure 9). The simulated task constraints gave the weighting matrix $W=\operatorname{diag}\left[\begin{array}{llllll}0 & 1 & 1 & 0 & 0 & 0\end{array}\right]$. The parameters for the experiment were set to: $\gamma=0.981 L_{f}=$ $1 \rho=150 \mathrm{~mm} \quad v_{t}=0.65 \mathrm{~m} / \mathrm{s}$. An obstacle was moving in a clockwise motion along the small red circle at $\sim 0.650 \mathrm{~m} / \mathrm{s}$. The outer red circle indicates the extent of the risk field $(\rho)$. The experiment was first ran along its planned path as a reference path, then with a classical potential field approach. The moving obstacle was designated a spherical potential field with radius $=\rho$. The resulting path from one pass can be seen as the yellow line. It is clear that the robot's end-effector did several sudden moves to avoid the frequently passing obstacle. The risk field was then enabled and the fast moving obstacle quickly built up a highly risky area along its path. The blue line in Figure 9 shows the resulting path with the risk field based control.

From the figure it can be seen that the new path for the robot is not only in a less risky area than that generated without the risk field, but it is also moving in a single curve away from its planned path. This single curve is less abrupt and may be experienced as a more comfortable path for the operator, further enhancing the trust. 
Table 3: Priority for Risk Reducing Measures

\begin{tabular}{cccccc}
\hline Control type & Max Risk & Reduction & Total Risk & Reduction & Time $[\mathrm{s}]$ \\
\hline Planned Path & 173.59 & - & 5751.47 & - & 13 \\
Obstacle avoidance & 150.67 & $13.2 \%$ & 4923.19 & $14.4 \%$ & 13 \\
Risk field based control & 80.72 & $53.5 \%$ & 2780.25 & $51.7 \%$ & 13 \\
\hline
\end{tabular}

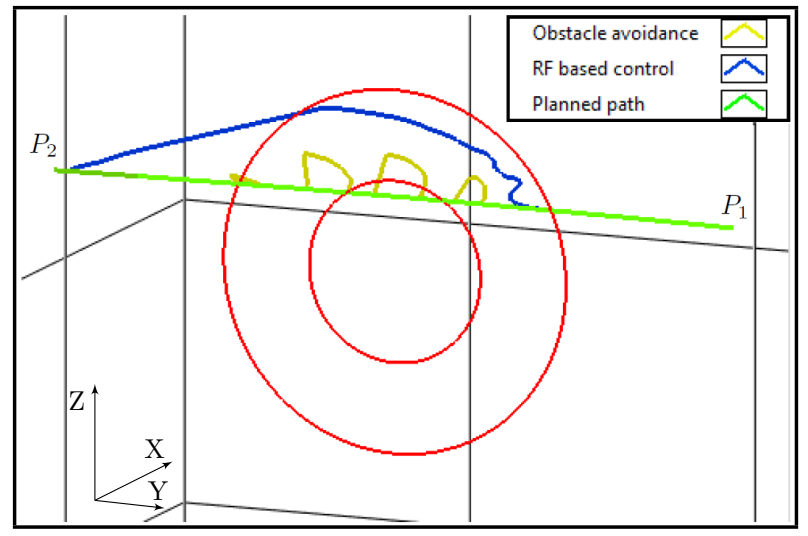

Figure 9: Paths taken by the robot with different control strategies.

We can also inspect the risk values throughout the paths. Using the risk field as an evaluation method, we can calculate the risk for the different paths. The risk level through time can be seen in Figure 10. The total and maximum risk through the path can be seen in Table 3

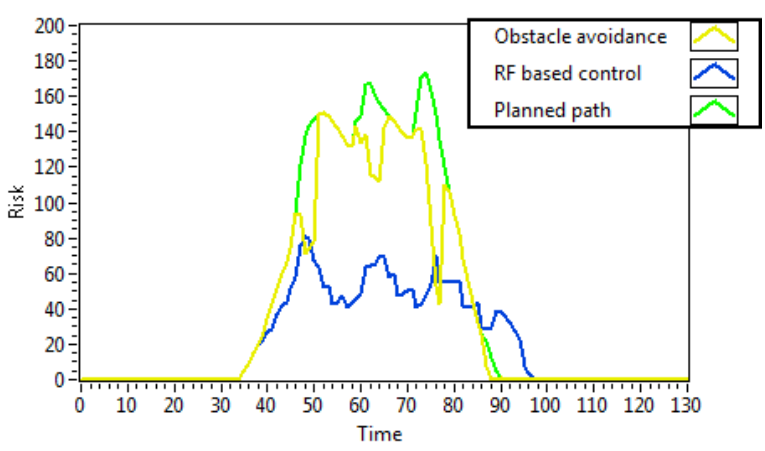

Figure 10: Risk through time for the different approaches.

It is clear that the general obstacle avoidance strategy barely reduces the total and maximum risk compared to the planned path, $13.2 \%$ and $14.4 \%$ reduction respectively. A significant reduction is achieved using the risk field. Both the maximum risk and total risk are reduced more than $50 \%$ compared to the planned path. The task is completed ( $P_{2}$ is reached) in the same 13 seconds for both approaches. Note that the risk is a dimensionless number and must only be compared to other risk values gathered using the same parameter settings. From a control perspective the different magnitudes are absorbed by the parameter $k_{v}$ in (12). The total risk was in this experiment calculated with a sample time of $100 \mathrm{~ms}$.

\section{Conclusion}

In this paper we have presented proactive safety strategy for human robot collaboration. The strategy is based on a quantified measure of risk. We have identified different aproaches to Risk Reducing Measures in robotic installations and placed our strategy amongst these. A new approach to safe human-robot collaboration was introduced using a risk field. The risk field is based on an analysis of the human's movement and the consequence of a collision with different human limbs, combined with a likelihood analysis. The risk field can be used both in a control strategy, and to evaluate a robot's path with regard to safety. Further, the risk field based safety strategy is proactive, which can improve the operators comfort of working alongside a robot. The building and degeneration of the risk field were simulated with a set of different parameters. The Risk Field was then implemented on a NACHI MR20 7-axes industrial robot. Although only one experiment were conducted, the method proved to be an efficient approach to reduce the risk. Compared to a classical obstacle avoidance approach, the risk through the path was significantly reduced.

\section{Acknowledgments}

The research was sponsored by The Norwegian Research Council and PPM AS through the Industrial PhD program.

\section{References}

Ahuactzin, J. M. and Gupta, K. K. The kinematic roadmap: A motion planning based global approach 
for inverse kinematics of redundant robots. IEEE Transactions on Robotics and Automation, 1999. 15:653-669. doi:10.1109/70.781970.

Balan, L. and Bone, G. M. Real-time 3D collision avoidance method for safe human and robot coexistence. In IEEE International Conference on Intelligent Robots and Systems. pages 276-282, 2006. doi:10.1109/IROS.2006.282068.

Behnisch, K. White Paper Safe collaboration with ABB robots Electronic Position Switch and SafeMove. 2008. pages 1-45.

Berenson, D. and Kuffner, J. Task Space Regions : A Framework for Pose- Constrained Manipulation Planning. Robotics Institute, 2011. Paper 1031. URL http://repository.cmu.edu/robotics/1031.

Chesbrough, H. W. The Era of Open Innovation. MITSloan Management Review, 2003. 44:35-41. doi:10.1371/journal.pone.0015090.

Galambos, P. Vibrotactile Feedback for Haptics and Telemanipulation : Survey, Concept and Experiment. Acta Polytechnica Hungarica, 2012. 9(1):4165.

Guo, Z. and Hsia, T. Joint trajectory generation for redundant robots in an environment with obstacles. In Proceedings., IEEE International Conference on Robotics and Automation, volume 1. pages 157-162, 1990. doi:10.1109/ROBOT.1990.125964.

Gupta, K. Path planning with general end-effector constraints: using task space to guide configuration space search. 2005 IEEE/RSJ International Conference on Intelligent Robots and Systems, 2005. pages 1875-1880. doi:10.1109/IROS.2005.1545305.

Haddadin, S. and Albu-Schaffer, A. Evaluation of Collision Detection and Reaction for a Human-Friendly Robot on Biological Tissues. 6th IARP/IEEERAS/EURON Workshop on Technical Challenges for Dependable Robots in Human Environments, 2008.

Haddadin, S., Albu-Schaffer, A., Frommberger, M., and Hirzinger, G. The DLR Crash Report : Towards a Standard Crash-Testing Protocol for Robot Safety - Part I : Results. In IEEE Intl. Conf. on Robotics and Automation. pages 272-279, 2009a. doi:10.1109/ROBOT.2009.5152602.

Haddadin, S., Albu-Schaffer, A., Frommberger, M., and Hirzinger, G. The DLR Crash Report: Towards a Standard Crash-Testing Protocol for Robot Safety - Part II : Discussions. In IEEE International
Conference on Robotics and Automation. pages 280287, 2009b. doi:10.1109/ROBOT.2009.5152711.

Hoffman, R. R., Johnson, M., Bradshaw, J. M., and Underbrink, A. Trust in Automation. Intelligent Systems, IEEE, 2013. 28(1):84-88. doi:10.1109/MIS.2013.24.

Khatib, O. Real-Time Obstacle Avoidance for Manipulators and Mobile Robots. In Robotics and Automation. Proceedings. 1985 IEEE International Conference on. pages 500-505, 1985. doi:10.1109/ROBOT.1985.1087247.

Koppula, H. S. and Saxena, A. Anticipating Human Activities using Object Affordances for Reactive Robotic Response. In Proceedings of Robotics: Science and Systems. 2013.

KUKA. KUKA Lightweight Robot. 2014. URL http://www.kuka-labs.com/en/service_ robotics/lightweight_robotics/.

Kulić, D. and Croft, E. a. Real-time safety for humanrobot interaction. Robotics and Autonomous Systems, $2006 . \quad 54(1): 1-12$. doi:10.1016/j.robot.2005.10.005.

Lacevic, B. and Rocco, P. Kinetostatic danger field - a novel safety assessment for human-robot interaction. In 2010 IEEE/RSJ International Conference on Intelligent Robots and Systems. IEEE, pages 2169-2174, 2010. doi:10.1109/IROS.2010.5649124.

Lacevic, B., Rocco, P., and Zanchettin, A. M. Safety Assessment and Control of Robotic Manipulators Using Danger Field. IEEE Transactions on Robotics, 2013. 29(5):1257-1270. doi:10.1109/TRO.2013.2271097.

Latombe, J.-C. and Barraquand, J. Robot Motion Planning: A Distributed Representation Approach. The International Journal of Robotics Research, 1991. 10(6):628-645. doi:10.1177/027836499101000604.

Mainprice, J. and Berenson, D. Human-robot collaborative manipulation planning using early prediction of human motion. 2013 IEEE/RSJ International Conference on Intelligent Robots and Systems, 2013. pages 299-306. doi:10.1109/IROS.2013.6696368.

MRK-Systeme. KR 5 SI (SafeInteraction) Prospect. 2014. URL www.mrk-systeme.de_downloads_ Prospekt_KR_5_SI_en.

Muramatsu, Y., Niitsuma, M., and Thomessen, T. Building a cognitive model of tactile sensations 
based on vibrotactile stimuli. 2013 IEEE 4th International Conference on Cognitive Infocommunications (CogInfoCom), 2013. pages 149-154. doi:10.1109/CogInfoCom.2013.6719231.

Nakabo, Y. and Ishikawa, M. Visual impedance using $1 \mathrm{~ms}$ visual feedback system. In Proceedings. 1998 IEEE International Conference on Robotics and Automation (Cat. No.98CH36146), volume 3. IEEE, pages 2333-2338, 1998. doi:10.1109/ROBOT.1998.680671.

NORSOK. NORSOK STANDARD Z-013N. September. 2001.

Oriolo, G., Ottavi, M., and Vendittelli, M. Probabilistic motion planning for redundant robots along given end-effector paths. In IEEE/RSJ International Conference on Intelligent Robots and Systems, volume 2. pages 1657-1662, 2002. doi:10.1109/IRDS.2002.1043993.

Patel, R. and Shadpey, F. Control of Redundant Robot Manipulators. Springer, 2005.

Petrič, T. and Žlajpah, L. Smooth continuous transition between tasks on a kinematic control level: Obstacle avoidance as a control problem. Robotics and Autonomous Systems, 2013. 61(9):948-959. doi:10.1016/j.robot.2013.04.019.

RethinkRobotics. Rethink Robotics. 2013. URL www . rethinkrobotics.com.

RIA/ANSI. R15.06 1999 American Standard for industrial Robots Safety Requirement. 1999.

Sanderud, A. R. and Thomessen, T. Releasing the Synergy of Human-Robot Collaboration - Redundant Robotics in Practice. ACTA Tehnica Corviniensis - Bulletin of Engineering, 2014. 7(1):161-164.

Sanderud, A. R., Thomessen, T., Hashimoto, H., Osumi, H., and Niitsuma, M. An approach to path planning and real-time redundancy control for human-robot collaboration. In 2014 IEEE/ASME International Conference on Advanced Intelligent Mechatronics. IEEE, pages 1018-1023, 2014. doi:10.1109/AIM.2014.6878214.

Sanderud, A. R. n. Task programming of Redundant Industrial Robots - A Virtually Extended Nullspace
Formulation Verified Through Obstacle Avoidance. Master's thesis, Norwegian University of Science and Technology, 2012.

Slovic, P. Perception of Risk. Science, 1987. 236(4799):280-285. doi:DOI: 10.2307/1698637.

SMErobotTM. The European Robot Initiative for Strengthening the Comprehensiveness of SMEs in Manufacturing. 2013. URL www.smerobot.org.

Stilman, M. Task constrained motion planning in robot joint space. In 2007 IEEE/RSJ International Conference on Intelligent Robots and Systems. IEEE, pages 3074-3081, 2007. doi:10.1109/IROS.2007.4399305.

Takimoto, A., Hashimoto, H., and Niitsuma, M. Effective destination determination for Semi-Autonomous Smart Electric Wheelchair Based on History of $\mathrm{Hu}-$ man Activity. In Industrial Informatics (INDIN), 2014 12th IEEE International Conference on. pages 769-775, 2014. doi:10.1109/INDIN.2014.6945609.

Thomessen, T. and Niitsuma, M. Cognitive HumanMachine Interface with multi- modal man-machine communication. In 4th IEEE International Conference on Cognitive Intocommunications. pages 873$876,2013$.

Tsuji, T., Akamatsu, H., and Kaneko, M. Noncontact impedance control for redundant manipulators using visual information. In Proceedings of International Conference on Robotics and Automation, volume 3. pages 2571-2576, 1997. doi:10.1109/ROBOT.1997.619348.

Zhang, Y. and Wang, J. Obstacle Avoidance for Kinematically Redundant Manipulators Using Dual Neural Network. IEEE Transactions on Systems Man and Cybernetics, 2004. 34(1):752-759. doi:10.1109/TSMCB.2003.811519.

Zlajpah, L. and Nemec, B. Kinematic control algorithms for on-line obstacle avoidance for redundant manipulators. In IEEE/RSJ International Conference on Intelligent Robots and Systems, volume 2. pages 1898-1903, 2002. doi:10.1109/IRDS.2002.1044033. 